\title{
Social and cultural barriers in the health assistance to indigenous people
}

\begin{abstract}
This paper briefly discusses the relevance of identifying social and cultural barriers in the health assistance to indigenous people of Argentina. Sustained in ethnographic fieldwork in an urban indigenous community of Argentina, the author wants to demonstrate the importance of the traditional conceptions and practices of the social group in order to interpret obstacles in the health interventions, not always considered. Also, it is important to attend to the social barriers related to the relations between social groups, expressed in discriminative and racism actions from the health services that discourage the population to be served. To guarantee the right to health, it is necessary to understand the complex logic that direct the actions of social groups.
\end{abstract}

Keywords: social barriers, cultural barriers, indigenous people, health care
Volume 5 Issue 3 - 2018

\author{
Stella M Orzuza \\ Rosario Institute of Research in Education Sciences (IRICE) \\ - National Council of Scientific and Technical Research \\ (CONICET), School of Psychology, Argentina
}

\begin{abstract}
Correspondence: Stella Orzuza, Rosario Institute of Research in Education Sciences (IRICE) - National Council of Scientific and Technical Research (CONICET), School of Psychology, National University of Rosario (UNR), Ocampo and Esmeralda, Rosario, Santa Fe, Argentina, Tel (54)(34I)482 I769/70, Email stella.m.orzuza@gmail.com
\end{abstract}

Received: January 23, 2018 | Published: May 04, 2018

\section{Introduction}

The purpose of this article is to demonstrate the relevance of identifying social and cultural barriers in the health assistance to indigenous people of Argentina. From the ethnographic work I have done with an indigenous community, I want to demonstrate how a series of processes which happen during the health attention to this people, could not be explained and interpreted without the consideration of their conceptions and practices. I want to highlight the importance of including it in health research and, fundamentally, in making diagnoses for future intervention in health education programs. Indigenous peoples and other social groups conceive and interpret the world and the body in different ways, derived from their particular way of appropriating nature and, therefore, generating a worldview. The experience of the individual illness is constructed and interpreted according to the shared worldviews, just as the social relations between groups constitute obstacles or reasons for interrupting treatments when they are materialized in unequal relationships. Therefore, in order to understand health-disease processes, particularly in areas of inter-ethnic contact, we need to know the ways in which they personally interpreted this process and analyze the role played by discrimination and racism in the healthdisease-care processes.

\section{Social and cultural barriers in health-disease-care process of rheumatic diseases}

The analysis showed in this article came from ethnographic fieldwork done in the last decade (2007-2017) in an indigenous community in Rosario, Argentina. The study was carried out in the city of Rosario to identify the prevalence of this group of diseases, with three stages: epidemiological, anthropological and genetic. I was part of the second stage under the direction of Anthropologist Marcela Valdata. The results of the epidemiological stage published, ${ }^{1}$ indicate a higher prevalence of this group of rheumatic diseases in this social group compared to the general population.

This population has the characteristic of residing in an important urban center of Argentina, having migrated from their traditional area of residence (Gran Chaco) in search of better living conditions. Even when they identified Rosario as their home, the population mobility was frequent in this social group. Not only did they continue the migratory movement from Gran Chaco to Rosario, but also they made trips from Rosario to Gran Chaco, usually to stay for a short period of time and return. In the visits to the Gran Chaco, they usually bring clothes to their relatives and other needed objects and return with materials for making ceramics or weaving baskets or clothing. I believe that these close relations between communities promote the preservation and reproduction of the traditional indigenous practices. Religion is also an element that unites the community. The first ethnographic work I did on rheumatic diseases in indigenous communities was within the framework of a larger study conducted by the Latin American Group of rheumatic diseases in native peoples.

Some of the most relevant problems chosen for the local interventions were: delay in the consultation; lack of knowledge of the disease, and interruption of treatment. ${ }^{2}$ Based on the results, the implementation of health education interventions was defined. The first intervention was in health promotion workshops, from which the first cultural barrier was identified: the cultural non-representation of the disease. In the culture of the indigenous community under study, there was no representation of this disease. People diagnosed with any of these diseases recognized that possibly their parents or uncles or grandparents should have suffered from this disease, but they did not know that these symptoms, taken as a whole, constituted a pathology, so they did not seek consultation, or only reported some of the symptoms. They usually received only pain care. Their traditional doctors, shamans, did not know these diseases, so there was no treatment within the culture for them. During the workshops, a name was defined in the language of the indigenous community, sehuet nam ypynaqa, which can be translated into English as "pain in the bones". Thus, these diseases began to exist, to have a representation within the indigenous culture studied, because it began to have a way of being named. A deep knowledge about the linguistic differences identified in the indigenous community could be consulted. ${ }^{3}$

The second barrier I want to comment on is a social one, that was identified during the workfield of my Ph.D. thesis, which is about accessibility to health system by indigenous people. This social barrier 
refers to the relation between the indigenous social group and the mayor society. One of the reasons for non-consultation in the hospital, told by the indigenous people, was "to be afraid". Generally, this affirmation is accompanied by a smile which showed embarrassment. With a deeper conversation about the reason of feeling fear, the people told narratives about negative experiences with the health system, which happened to them or relatives. Experiences of discrimination and racism, like making them wait until everyone non-indigenous had been attended; criticism of the ways of raising their children based on prejudices, scolding and being ridiculed during the attention. A different behavior is shown in the Primary Care Center, where the indigenous people have a trust relationship with the doctors, where this barrier seems to had been overcome. Unfortunately, this is not an unknown barrier. A specialist in equity on health, ${ }^{4}$ pointed out that any consideration about social inequalities in ethnic groups has a double determination: the social position of this particular group in the society and the acceptance or rejection in front of the majority groups. The discrimination practiced against certain ethnic groups is a way of expressing and institutionalizing social relations of domination and repression.

The third barrier I want to point to is the "healing by faith". The religion is an indispensable element to be investigated in healthdisease processes in excluded populations. ${ }^{5}$ During the years of ethnographic work that I have done in this community, I have learned that many of them had joined the evangelical religion. They used to consult with the leaders of the church for healing as well as ask for prayer during the religious ceremony. However, having established relationships of trust with the community, I was able to know that some of them, when they did not find the healing of the disease at the public health system, abandoned the treatments to surrender to the miraculous cure. The problem with these types of diseases, is that the interruption of the treatment allows the disease to advance. A previous study, ${ }^{5}$ identified that, in an evangelical gypsy community, the new religion that the community adopts (although in some points it contradicts its ethnic identity) reinforced the use of the public health system, granting it a divine recognition while generating a rejection of traditional medicine. It reaffirms what was said in a recent study, ${ }^{6}$ in which the authors pointed out in a bibliographical review of facilitators and obstacles for the attention of chronic diseases in the native population, that what can be a facilitator in one community can be an obstacle in another.

\section{Conclusion and recommendation}

The cultural and social barriers identified and briefly described in this article explain the importance of addressing both the traditional conceptions and practices of indigenous populations because they could be functioning as barriers to attention, as well as situations of discrimination and racism that discourage the population to be served. It is also important to identify cultural facilitators, both in terms of traditional practices and indigenous leaders who can be allies in the care, or facilitate communication as translators. Cultural diversity implies challenges for health care. Respect for their traditional practices and conceptions cannot neglect the identification of practices and knowledge that may threaten the care of their health. From an effective dialogue and relationships of trust, it is possible to attend to situations by looking for alternatives that are beneficial for all. Moving towards more equal relationships would need to overcome the social barriers that reproduce the existing inequalities in society. In relation to religious practices and their possibilities of attacking biomedical treatments, we are devising community interventions, with community agents, pastors of the evangelical religion who attend health care providers, asking them to encourage their parishioners to not abandon the treatments, even if they do not achieve the expected results.

The cultural diversity present in our societies, the complex web of identities that are woven in our territory, make it necessary, in a situated understanding of health problems, to address the conceptions of those who suffer, about their suffering. Health and illness are lived in frames of meaning that determine the procedures of sufferers. To guarantee the right to health, it is necessary to understand the complex logic that directs the actions of social groups.

\section{Acknowledgements}

None.

\section{Conflict of interest}

None.

\section{References}

1. Quintana R, Silvestre AM, Goñi M, et al. Prevalence of musculoskeletal disorders and rheumatic diseases in the indigenous Qom population of Rosario, Argentina. Clin Rheumatol. 2016;35(Suppl 1):5-14.

2. Fernández S, Orzuza S, Valdata $M$, et al. Therapeutic practices in the treatment of rheumatic diseases in the Qom population. Rosario, Argentina, XI Argentine Conference of Social Anthropology. 2014.

3. Valdata M, Orzuza S, Fernández S, et al. Diagnosis and evaluation of results of university extension workshops on rheumatoid arthritis in the Qom population. Santa Rosa, Argentina, II International Conference Indigenous People of Latin America, S. XIX-XXI. 2016.

4. Barradas Barata R. Do ethnic misrepresentations necessarily mean racism? How and Why Social Inequalities are harmful to Health. Brazil, Fiocruz, Rio de Janeiro: 2009. p. 55-73.

5. Ramirez Hita S. Health, ethnicity, and religion. Health in excluded populations. Notebooks of the Catalan Institute of Anthropology. 2006;22:101-116.

6. Gibson O, Lisy K, Davy C, et al. Enablers and barriers to the implementation of primary health care interventions for Indigenous people with chronic diseases: a systematic review. Implementation Science. 2015;10(71):2-11. 